\title{
Single-cell transcriptomics of murine mural cells reveals cellular heterogeneity
}

\author{
Ya-Na Guan ${ }^{1 \dagger}$, Yue $\mathrm{Li}^{2 \dagger}$, Moom Roosan ${ }^{2 *} \&$ Qing Jing ${ }^{1 *}$ \\ ${ }^{1}$ Shanghai Jiao Tong University School of Medicine (SJTUSM) \& CAS Key Laboratory of Tissue Microenvironment and Tumor, Shanghai \\ Institute of Nutrition and Health, Chinese Academy of Sciences (CAS), Shanghai 200031, China; \\ ${ }^{2}$ Chapman University, Irvine, CA 92618, USA
}

Received July 30, 2020; accepted September 23, 2020; published online November 3, 2020

\begin{abstract}
Mural cells (MCs) wrap around the endothelium, and participate in the development and homeostasis of vasculature. MCs have been reported as heterogeneous population morphologically and functionally. However, the transcriptional heterogeneity of MCs was rarely studied. In this study, we illustrated the transcriptional heterogeneity of MCs with different perspectives by using publicly available single-cell dataset GSE109774. Specifically, MCs are transcriptionally different from other cell types, and ligand-receptor interactions of different cells with MCs vary. Re-clustering of MCs identified five distinct subclusters. The heterogeneity of MCs in tissues was reflected by MC coverage, various distribution of MC subclusters, and ligand-receptor interactions of MCs and parenchymal cells. The transcriptomic diversity of MCs revealed in this article will help facilitate further research into MCs.
\end{abstract}

mural cells, single-cell analysis, heterogeneity

Citation: Guan, Y.N., Li, Y., Roosan, M., and Jing, Q. (2021). Single-cell transcriptomics of murine mural cells reveals cellular heterogeneity. Sci China Life Sci 64, 1077-1086. https://doi.org/10.1007/s11427-020-1823-2

\section{INTRODUCTION}

Mural cells (MCs), which support the structure of vessels and play vital roles in maintaining vascular homeostasis (Armulik et al., 2011), mainly consist of vascular smooth muscle cells (VSMCs) and pericytes (PCs) in vasculature. MCs are ubiquitously distributed along the abluminal endothelium and the phenotype varies with the hierarchically distributed microvascular tree, which contains arterioles, precapillary arterioles, capillaries, postcapillary venules, and venules (Hartmann et al., 2015). Specifically, PCs extend along the capillary bed with nearly rounded cell bodies and their derived cytoplasmic processes (Armulik et al., 2005; Borysova et al., 2013). VSMCs cover larger blood vessels

$\dagger$ Contributed equally to this work

*Corresponding authors (Moom Roosan, email: roosan@chapman.edu; Qing Jing, email: qjing@sibs.ac.cn) with different morphology (Rensen et al., 2007). Generally, arteriolar endothelium-encircled VSMCs are spindle-shaped, tightly arranged cells without cytoplasmic process. By contrast, VSMCs along the venules are bigger, stellate-shaped, with many slender, branching cytoplasmic processes. The phenotype of mural cells in precapillary arterioles and postcapillary venules gradually transits from VSMCs to PCs and vice versa.

The diversity of mural cells extends beyond the phenotypes mentioned above. In fact, the ultrastructure and function of MCs in different tissues are diverse, presumably to meet the distinct physiological need (Augustin and Koh, 2017). For instance, MCs in the brain are densely branched and regulate the permeability (Daneman et al., 2010), angiogenesis (Arnold et al., 2014) and neuroinflammatory (Kovac et al., 2011) responses of the blood-brain barrier (BBB). While cardiac MCs, which have long processes 
ubiquitously wrapping the continuous endothelium, regulate blood flow and communicate with the myocardial microvasculature (Nees et al., 2012). Cardiac pericyte was also recently reported as potential mechanism of heart injury infected by SARS-CoV-2, due to its relatively higher expression of ACE2 (Chen L. et al., 2020). Furthermore, MC abundance, which was reflected by the ratio of MCs and ECs, is tissue-dependent. The central nervous system (CNS) microvasculature was reported as the highest MC coverage, with a ratio of 1:1 to 1:3 (Mathiisen et al., 2010; Armulik et al., 2010). The lowest MC coverage up to now was stated as human skeletal muscle, with a ratio of 1:100 (Shepro and Morel, 1993). The degree of MC abundance is highly related to the activity of ECs in the tissue, blood pressure, and the barrier properties of blood-tissue, such as blood-brain barrier in CNS, blood-air barrier in the lung (Diaz-Flores et al., 2009; Sims et al., 1994; Kato et al., 2018). Any abnormality of MCs in structure or abundance may lead to diseases, such as diabetes, cancer or other diseases related with vascular instability (Winkler et al., 2018; Cooke et al., 2012; Hinkel et al., 2017).

With the development of sequencing methods, plenty of genes were reported to be highly expressed in MCs (Table S1 in Supporting Information), which facilitates their further exploration. However, controversy still remains, mainly due to lack of sensitive, specific molecular marker for MCs (Chen W. C. et al., 2015; Guimarães-Camboa et al., 2017). Besides, the heterogeneity of MCs in transcriptomics was also rarely studied, despite the known diverse functions of MCs.

In this article, we explored the transcriptional diversity of MCs, leveraging publicly available single-cell dataset GSE109774 (Schaum et al., 2018). We found MCs are heterogeneous. Ligand-receptor interactions of MCs and other cell-types vary. Further-clustering of MCs identified five distinct subpopulations. The heterogeneity of MCs in tissues was reflected by MC coverage, the distribution of subpopulations and ligand-receptor interactions between MCs and parenchymal cells.

\section{RESULTS}

\section{MC coverage and interaction with other cells varied}

To delineate the transcriptional characteristics of MCs, all samples of published single-cell dataset GSE109774, which contains data of 20 tissues with two different sequencing methods (Schaum et al., 2018), were interrogated and analyzed at single-cell resolution. Eight out of 46 tissues were found to have cluster of MCs. As shown in Figure 1, cells in each tissue were divided and defined as different populations by differentially expressed genes (DEG) of each cluster (as listed in Table S2 in Supporting Information with marker genes highlighted). Percentage of each cluster was demonstrated by histogram, and ratios between $\mathrm{MC}$ and $\mathrm{EC}$ are summarized in Table 1. Obviously, MC coverage in different tissues varied. Among them, the MC coverage of bladder was the highest, with a ratio of 1:1.3, that is, almost every EC was covered by one MC on average. While pulmonary ratio was the lowest, with more than 20 ECs were covered by one MC. The MC coverage in the brain was quite consistent with reported studies, while the ratio in limb muscle was much higher than the reported 1:100 in skeletal muscle, which might be due to different sampling location.

Furthermore, we sought to perform unbiased ligand-receptor interaction analysis between MCs and other cell types by taking advantage of CellphoneDB (Vento-Tormo et al., 2018). As shown in Figure 1 and Table S3 in Supporting Information, MCs interact with almost every cell type. Overall, interactions of MCs and non-parenchymal cells, including ECs, fibroblasts, monocytes (include B cells, T cells, neutrophils, and macrophages) were similar in each tissue with different extent. Specifically, the receptor of CD74 on monocytes directs the strongest ligand-receptor interaction with MIF and APP produced by MCs (Figure S1 in Supporting Information). Most common interactions between fibroblasts and MCs were collagen-a1b1 complex, AXL-GAS6, PDGFA-PDGFRA, and LGALS9-CD44 (Figure S2 in Supporting Information). Interactions between ECs and MCs were studied most. Except canonical PDGFB-

Table 1 Mural cell coverage varies in each tissue

\begin{tabular}{ccccccc}
\hline Source tissue & Method & Total & EC & MC & MC:EC \\
\hline Bladder & Droplet & 2,500 & 33 & 695 & $1: 1.375$ \\
Brain & FACS & 3,106 & 1,247 & 111 & 24 & $1: 4.512987$ \\
Trachea & FACS & 4,513 & 1,247 & 221 & 55 & $1: 5.625$ \\
Heart & FACS & 2,782 & 392 & 1,339 & 161 & $1: 7.127273$ \\
Kidney & Droplet & 4,506 & 602 & 33 & $1: 8.31677$ \\
Muscle & Droplet & 4,570 & 710 & 30 & $1: 18.24242$ \\
Fat & FACS & 1,681 & & $1: 23.66667$ \\
Lung & FACS & & &
\end{tabular}



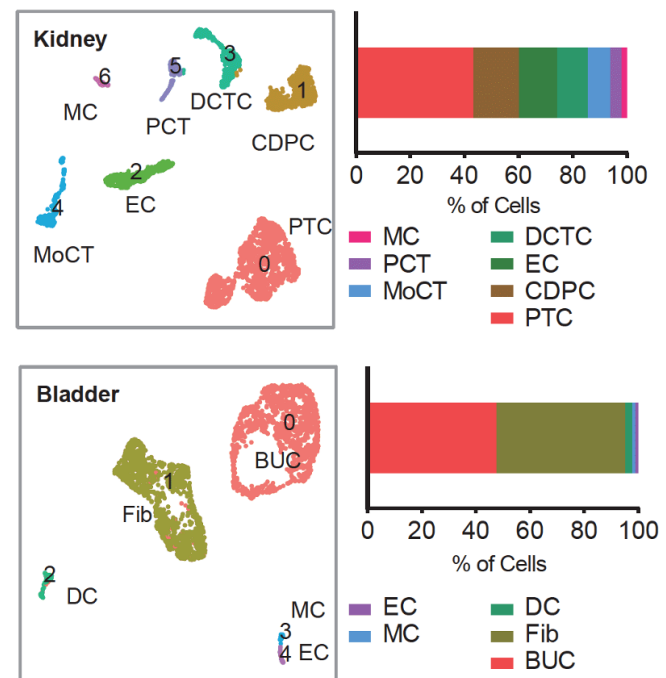

C

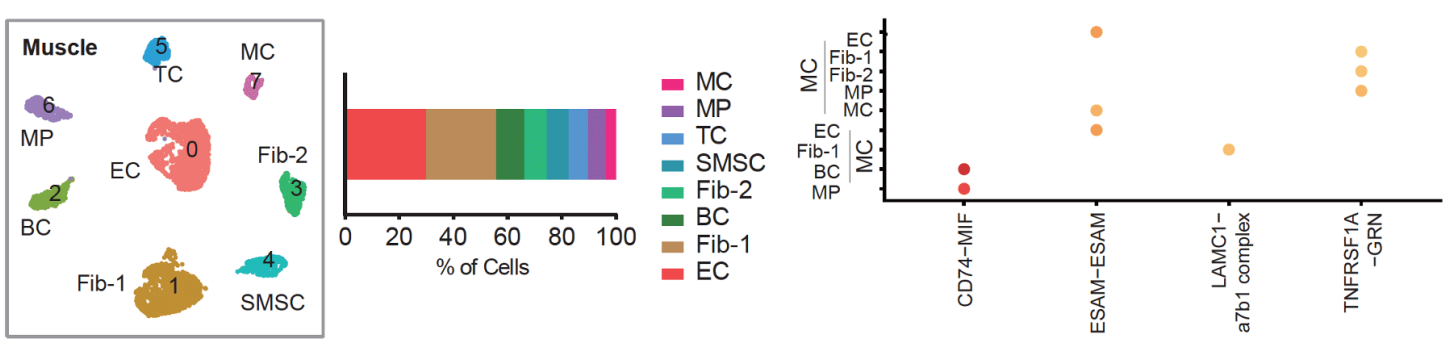

D
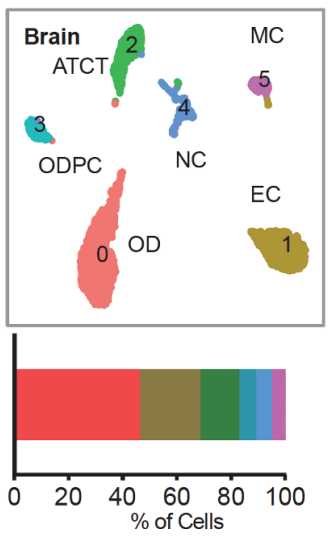

- MC $=$ ODPC $=\mathrm{EC}$

- NC $\square$ ATCT $\square$ OD

E

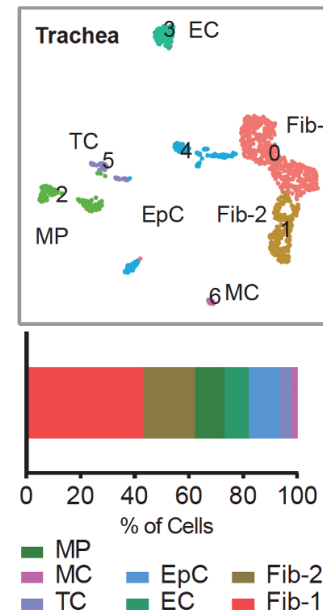

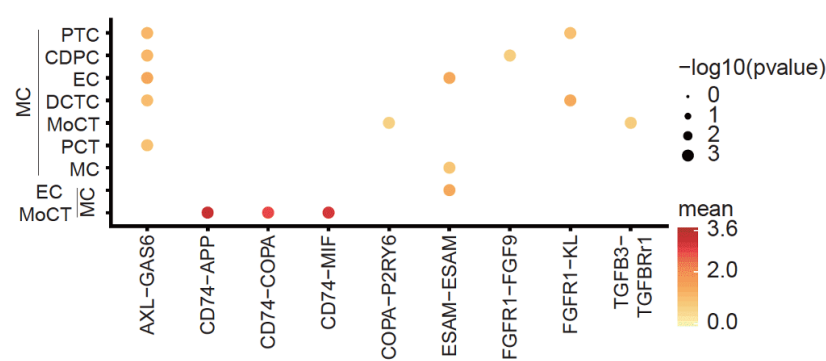
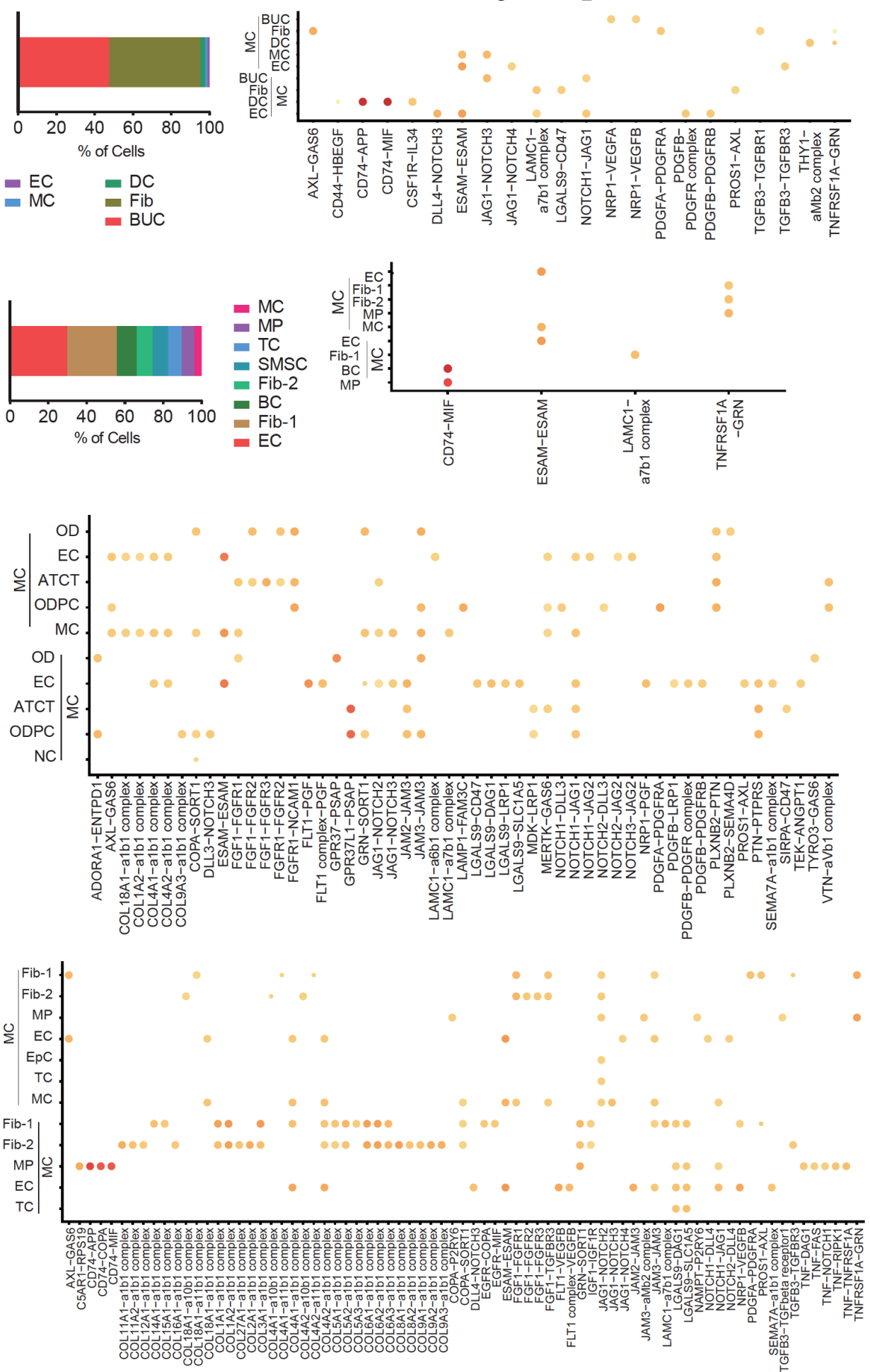

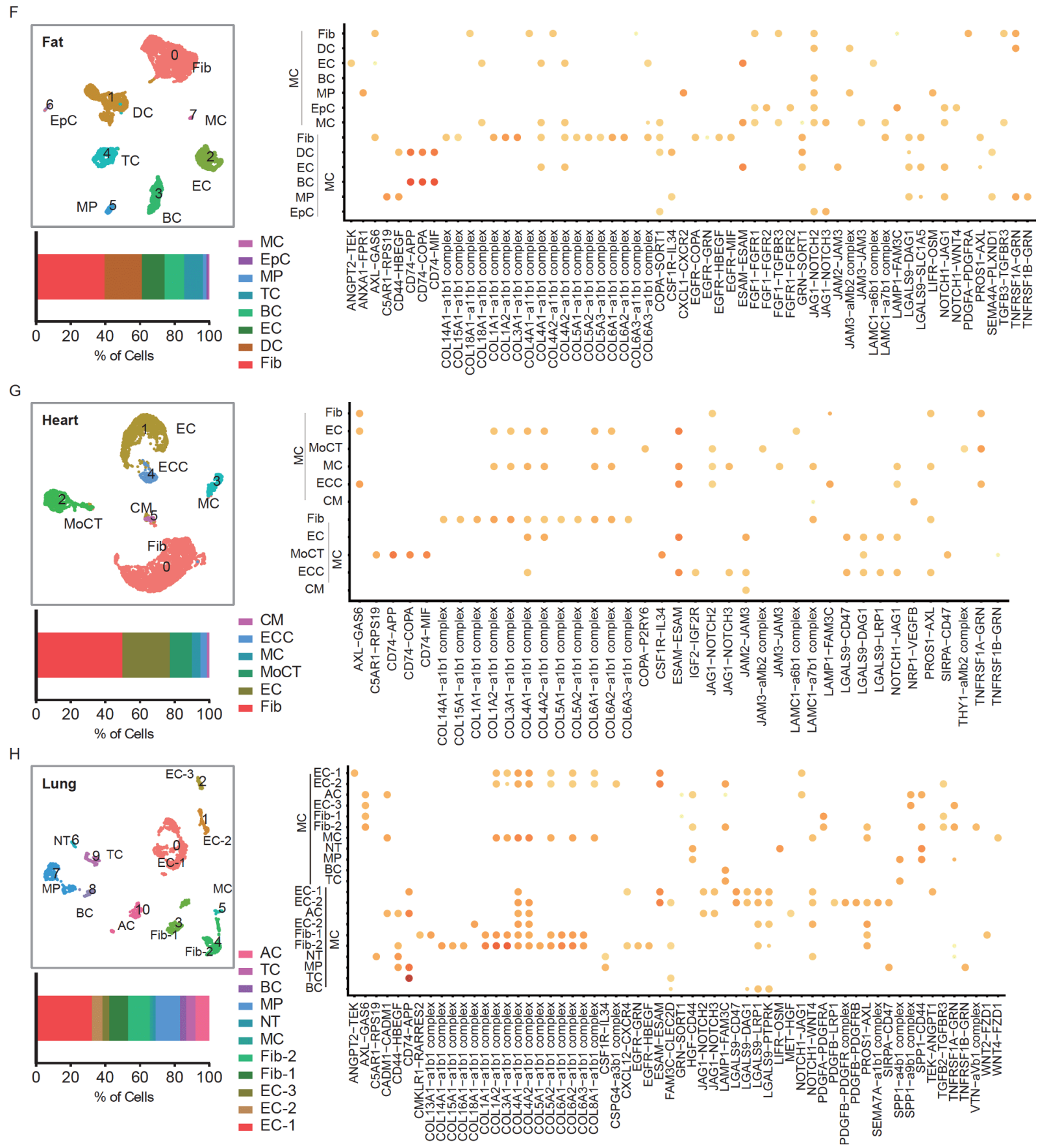

Figure 1 Single-cell clustering and ligand-receptor analysis in samples of published datasets GSE109774. Single-cell clustering and ligand-receptor analysis were performed in the kidney (A), bladder (B), muscle (C), brain (D), trachea (E), fat (F), heart (G), and lung (H) tissues. The distribution of each cluster was demonstrated by histogram. Definition of each cluster was based on established markers. Different colors denote different cell types. EC: endothelial cell; MC: mural cell; Fib: fibroblast; MoCT: monocyte; TC: T cell; BC: B cell; MP: macrophage; DC: dendritic cell; NT: neutrophil; EpC: epithelial cell; PCT: podocyte; DCTC: distal convoluted tubule cell; CDPC: collecting duct principal cell; PTC: proximal tubule cell; BUC: bladder urothelial cell; NC: neuron cell; OD: oligodendrocyte; ODPC: oligodendrocyte precursor cell; ATCT: astrocyte; SMSC: skeletal muscle satellite cell; CM: cardiomyocytes; ECC: endocardial cell; AC: alveolar cell.

PDGFRB, we found ECs interacted with MCs by secreting LGALS9 (Figure S3 in Supporting Information). Besides, interactions of MCs and parenchymal cells (except ubiquitously distributed cells like ECs, fibroblast, and monocyte) were more tissue-specific (Figure S4 in Supporting In- formation). For instance, MCs communicated with bladder urothelial cells (Figure 1B) and cardiomyocytes (Figure 1G) by NRP-VEGF interactions. Interactions of PLXNB2-SEMA4D, GPR37-PSAP, and GPR37L1-PSAP (Figure 1D) were brain-specific. NOTCH2-JAG1 and MET-HGF were 
restricted to the trachea (Figure 1E) and the lung (Figure 1H), respectively.

Collectively, MC coverage and ligand-receptor interactions were diverse in tissues with different extent.

\section{Mural cells are transcriptionally different from other cell types}

Next, eight tissues selected above were aggregated computationally to facilitate the heterogeneity analysis of MCs at the transcriptional level. As shown in Figure 2A, cells of the integrated results can be divided into six distinct populations, including EC (cluster 1, Pecam $1^{+} \mathrm{Cdh} 5^{+}$), fibroblast (cluster $\left.0, \mathrm{Pdgfra}^{+} \mathrm{Colla1}^{+}\right), \mathrm{MC}$ (cluster $10, \mathrm{Myh}_{1} 1^{+} \mathrm{Acta}^{+} /$ Pdgfrb $^{+} \mathrm{Cspg} 4^{+}$), macrophage (cluster 2, $\mathrm{Csf}_{2} \mathrm{ra}^{+} \mathrm{Cd} 14^{+}$), immune cell (cluster 3, including B cells of $\mathrm{Cd} 79 \mathrm{a}^{+} \mathrm{Cd} 19^{+}$, and $\mathrm{T}$ cells of $\mathrm{Cd} 2 \mathrm{~d}^{+} \mathrm{Lck}^{+}$), and parenchymal cell (cluster 49, cells within grey line). Gene ontology (GO) biological function (BP) analysis of DEG in MCs (Table S4 in Supporting Information) in Figure 2B was in agreement with reported potential of MCs in blood-flow control and angiogenesis (Peppiatt et al., 2006; Gerhardt and Betsholtz, 2003; Nehls et al., 1992; Teichert et al., 2017).

To evaluate reported mural cell biomarkers, expression profiles of genes listed in Table S1 in Supporting Information were plotted as shown in Figure $2 \mathrm{C}-\mathrm{G}$. Genes listed in
A

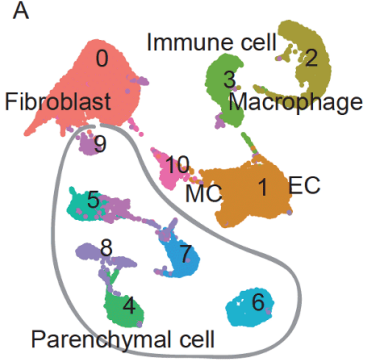

C

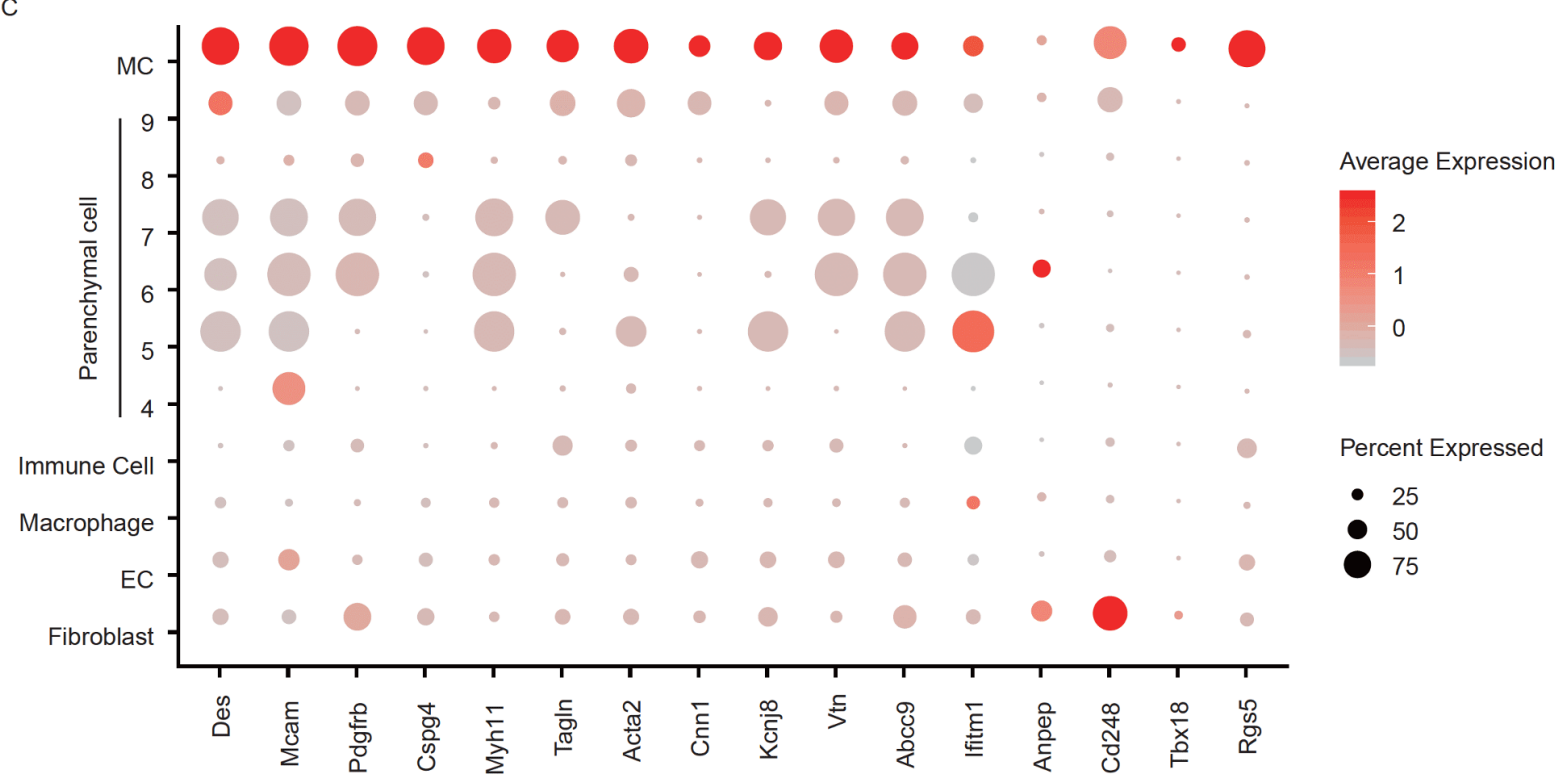

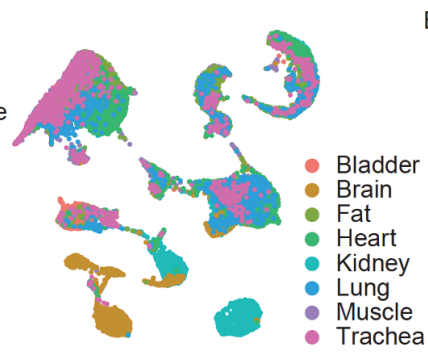

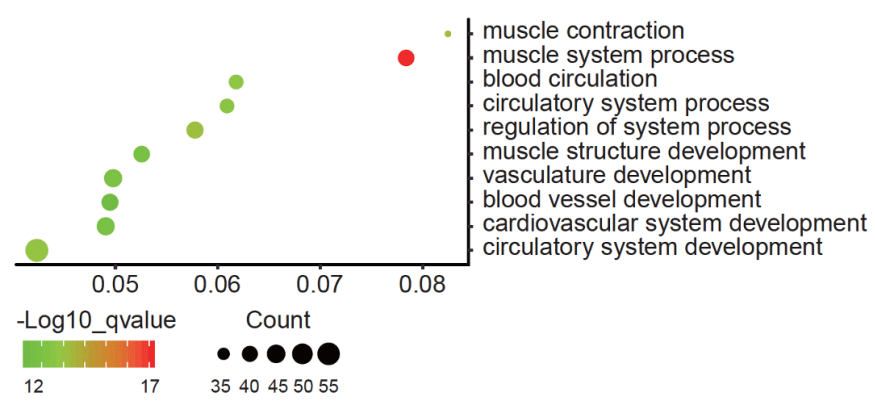

D

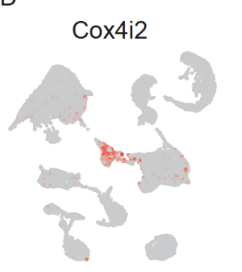

Figure 2 Integrative analysis of eight tissues from published datasets GSE109774.A, Cells from eight tissues were classified into 11 clusters. Left: different colors denote different cell types. Right: different colors denote different tissues. B, Gene ontology (GO) biological function (BP) analysis of differentially expressed genes (DEG) in cluster 10. C-G, Expression profiles of reported markers for MCs. H, Expression profiles of newly found marker genes for MCs. EC: endothelial cell; MC: mural cell; Fib: fibroblast; MP: macrophage. 
Figure 2C were highly but not exclusively expressed in MCs. Negative filters were necessary for them to mark MCs. The expression profiles of Mcam were consistent with reported studies that Mcam was highly expressed in ECs, MCs and some parenchymal cells in the brain (Gao et al., 2017; Chen J. et al., 2017). Pdgfrb was highly expressed in both MCs and Fibroblasts. Des was highly expressed in MCs and some muscle cells. The expression of Cspg4 in mural cells was lower than others, and was also expressed in parenchymal cells of the brain. Genes listed in Figure 2D and E were complementary to each other, and expressed in part of the mural cells. Considering the constitution of mural cells and gene functions, we assumed genes in Figure 2D and E were markers of VSMCs and PCs, respectively. Noticeably, most genes in Figure 2E were still not exclusively expressed in PC. Genes listed in Figure 2F were lowly expressed in mural cells, and not good enough to mark mural cells. Rgs5 was the best molecular marker for MCs in Table S1 in Supporting Information, due to its high specificity in expression (Figure $2 \mathrm{G})$. Furthermore, we offered some other potential genes relatively highly and exclusively expressed in MCs, as in Figure $2 \mathrm{H}$.

Conclusively, MCs were transcriptionally different from other cell types. Genes highly and exclusively identified in MCs will facilitate further exploration.

\section{Mural cells are transcriptionally heterogeneous}

To illustrate the heterogeneity of MCs at single-cell resolution, re-clustering was performed to MCs (cluster 10 in Figure 2A). As shown in Figure 3A, MCs from eight organs can be classified into five subclusters, of which four clusters were multi-tissue-derived, except cluster 4 , in which cells were derived from the lung, and we named it as cluster pulmonary MC (PMC). Figuring the widely accepted tissuespecific heterogeneity of ECs (Schaum et al., 2018; Paik et al., 2018; Kalucka et al., 2020) and re-clustering analysis by aggregation ECs of each tissue (Figure S5 in Supporting Information), we assumed that the heterogeneity of MCs in tissues was less than that of ECs.

To characterize other clusters, the expression profiles of genes marking VSMCs (Tagln, Myh11, Acta2), PCs (Kcnj8, Vtn, Abcc9), and ECs (Pecam1, Egfl7, Cdh5) in all MCs were plotted and analyzed. As shown in Figure 3B-D, markers of VSMCs were highly expressed in cluster 0 and cluster 2. Markers of PCs were highly expressed in cluster 1 and cluster 2. Markers of ECs were highly expressed in cluster 3. Based on the expression profiles, we assumed cluster 0 -cluster 3 as VSMC (cluster 0), PC (cluster 1), transitional mural cells between VSMCs and PCs (TMC, cluster 2), which express markers of both VSMCs and pericytes, and endothelial-like mural cells (EMC, cluster 3), which express markers of both MCs and ECs. Therefore, we assumed MCs are heterogeneous in transcriptome (Figure $3 \mathrm{E}$ ), which is partially consistent with their morphological distribution. Genes exclusively and highly expressed in each cluster are shown in Figure 3F by interrogating DEGs of each cluster (Table S5 in Supporting Information). Heatmap analysis of the top-10-ranking expressed gene sets performed by gene set variation analysis (GSVA) revealed that MCs in each cluster were involved in different biological processes (Figure 3G). Gene sets of VSMC and PC were indeed relatively enriched in TMC, consistent with the status of TMC. Furthermore, we analyzed the proportion of the five subclusters in each tissue. As shown in Figure $3 \mathrm{H}$, the ratio varied in each tissue, which was assumed to meet the tissuespecific vasculature.

\section{DISCUSSION}

As supporting cells in vasculature, endothelial cells (ECs) and mural cells (MCs) play vital roles in vascular homeostasis (Armulik et al., 2005). The heterogeneity of ECs in transcriptomics, structures, and functions have been wellstudied, especially with the development of the single-cell RNA sequencing (Kalucka et al., 2020; Nolan et al., 2013; Aird, 2007a, 2007b; Potente and Mäkinen, 2017). However, research about MCs was limited, potentially owing to (1) fewer MCs than ECs in organism; (2) difficulty of detachment MCs from vascular bed while isolating single cells; (3) lack of differentiation methods of MCs in vitro. With the development of next-generation and single-cell sequencing, several genes were declared to mark MCs, as listed in Table S1 in Supporting Information. Despite such advancement, controversy still exists on the heterogeneity and functions of MCs in various tissues.

In this article, we dissected the characteristics of MCs in different tissues by taking advantages of single-cell RNA analysis. To start with, we interrogated samples of eight tissues and revealed the diverse coverage and ligand-receptor interactions. Varied mural cellular coverage was assumed to meet different characteristics including blood-tissue barrier and EC turnover in each tissue. For instance, the high MC coverage in the bladder and brain indicates low-permeability and less passive transport of materials, which ensures the function of urine storage in the bladder and restricts the exchange of harmful substances in the brain. In fact, decreased MCs may lead to bladder tumors and arteriovenous malformation of the brain (O'Keeffe et al., 2008; Winkler et al., 2018). On the contrary, the low MC coverage in the lung facilitates oxygen metabolism of the alveoli. Excessive MCs are associated with pulmonary hypertension (Ricard et al., 2014). Various ligand-receptor interactions of MCs with other cells illustrated multiple functions. For instance, common interactions of MCs and non-parenchymal cells like 


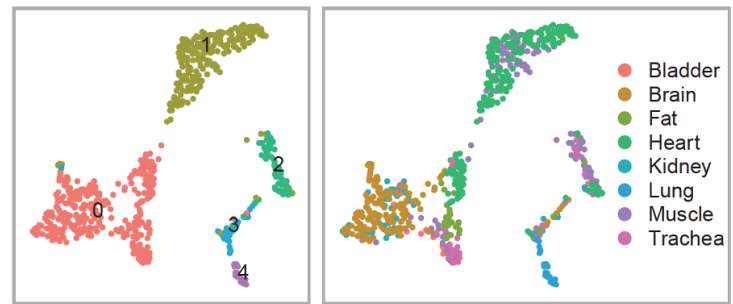

$E$

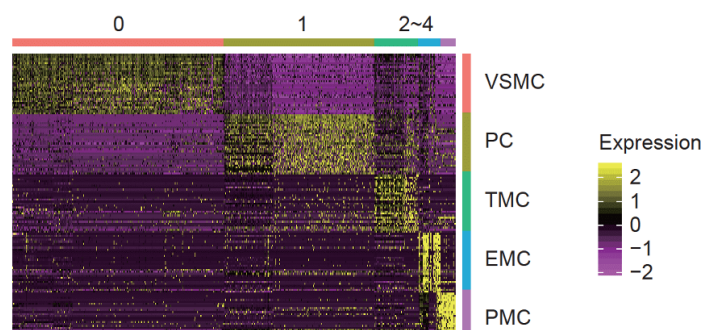

B

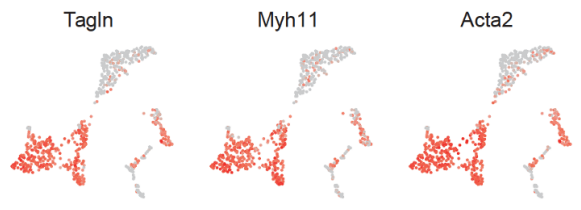

C
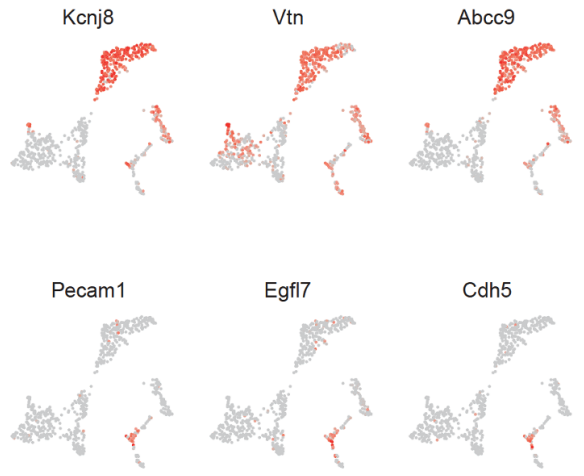

$\mathrm{F}$

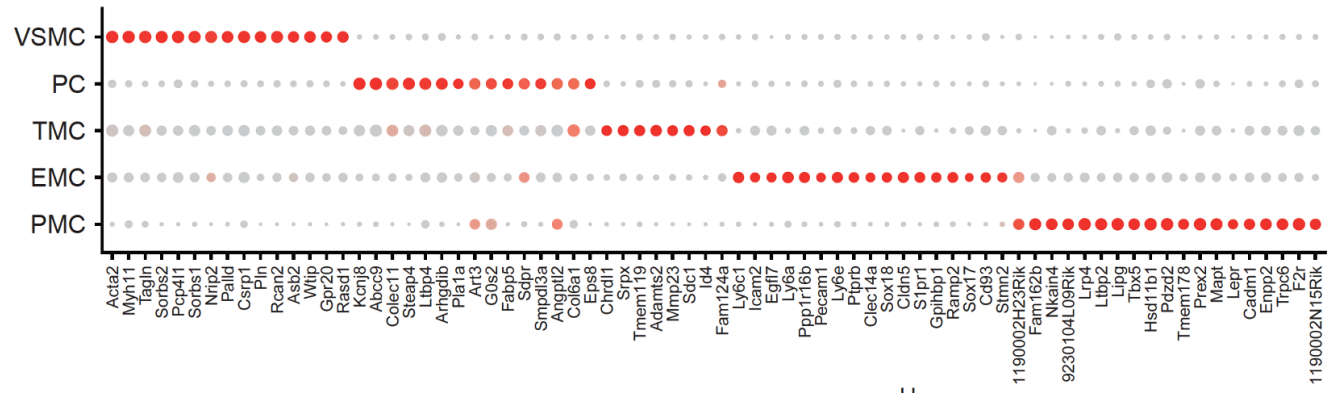

G

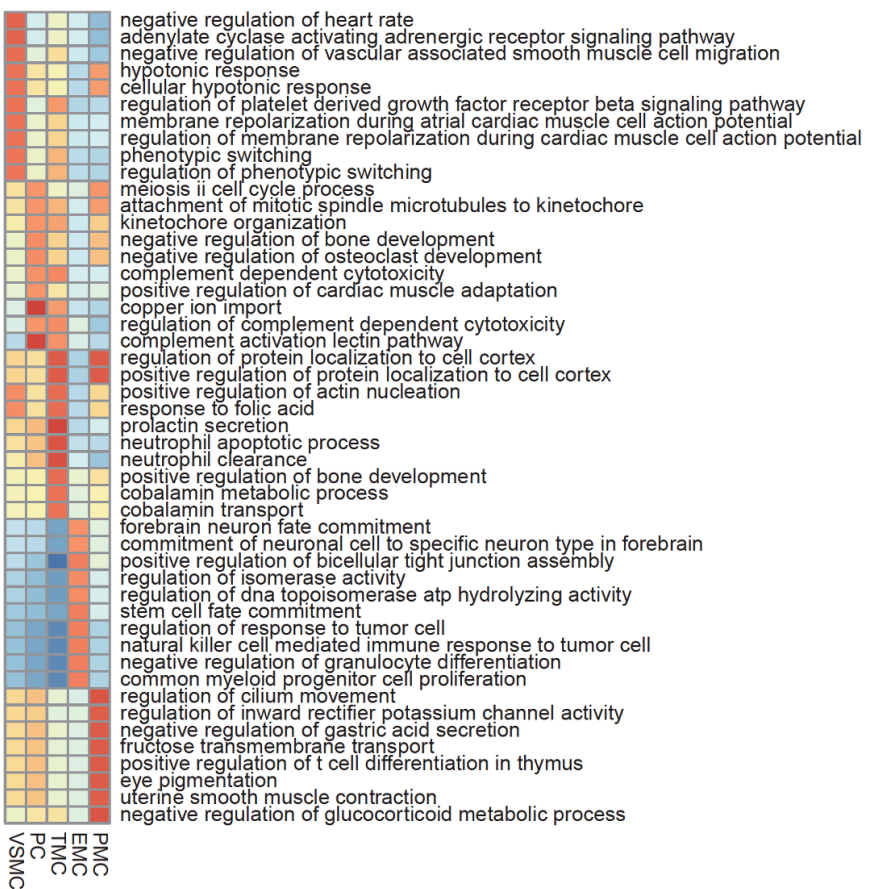

$\mathrm{H}$

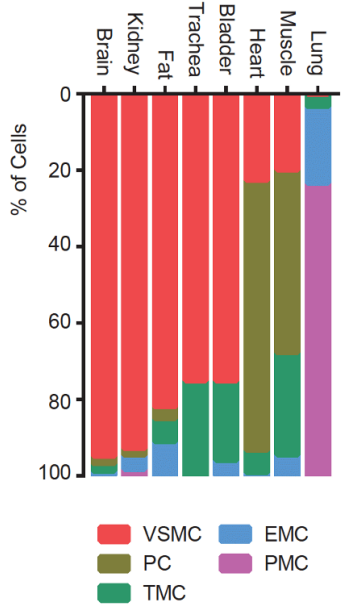

Figure 3 Cells defined as mural cells were heterogeneous. A, Re-clustering of mural cells identified five subclusters. Different colors denote different cell types (left). Different colors denote different tissues (right). B-D, Expression profiles of markers for vascular smooth muscle cells (B), pericytes (C), and endothelial cells (D). E, Heatmap of the top 20 highly expressed genes in each cluster of mural cells. Columns denote cells; rows denote genes. F, Genes highly and relatively exclusively expressed in each cluster of mural cells. G,Heatmap showing processes enriched in different clusters performed by gene set variation analysis (GSVA). H, Percentage of each cluster in different tissues varies. Different colors denote different tissues. VSMC: vascular smooth muscle cell; PC: pericyte; TMC: transitional mural cells between VSMCs and PCs; PMC: pulmonary specific mural cells; EMC: endothelial-like mural cells. 
monocytes, ECs, fibroblasts were consistent with potential functions of MCs, including immunoregulation and neuroregulation (Matsuda et al., 2009; Pantouris et al., 2018), cell migration and inflammatory processes (Wu et al., 2014; MacDonald et al., 2001; Shibata et al., 2020; Chou et al., 2013), vascular stability, and angiogenesis (Saban et al., 2008; Fantin et al., 2014). Tissue-specific interactions of MCs and parenchymal cells also reminded us of tissue-dependent potentials, like VSMC hyperplasia (Boucher et al., 2013), epithelial homeostasis in the alveolar (Jung et al., 2019).

Then, we sought to explore the mural cellar characteristics in transcriptome. We invested the uniqueness of MCs by comparing them with other cell types and integrating singlecell datasets of eight tissues. We also evaluated genes reported as MC markers, and offered some more genes highly and relatively specially expressed in MCs. All these marker genes will ultimately facilitate the illustration of function and mechanism in MCs. Re-clustering analysis of all MCs in each tissue indicated the heterogeneity of MCs. With the expression profiles of several marker genes, MCs can be divided into five heterogeneous subpopulations of VSMCs, PCs, EMCs (endothelial-like mural cells), TMCs (transitional mural cells between VSMCs and PCs), and PMCs (pulmonary mural cells), and the heterogeneous characteristic was consistent with the morphology of MCs. However, the tissue-specific heterogeneity of MCs was less than that of ECs, which may be due to the limited number of tissuespecific MCs in the analysis. Future studies need to include a larger number of MCs in single-cell analysis to facilitate the further identification of additional MC characteristics.

\section{MATERIALS AND METHODS}

\section{Data resources}

A publicly available single-cell sequencing dataset used in this study was downloaded from Gene Expression Omnibus (GEO) repository (GSE109774). The dataset contains two different sequencing methods-FACS and Droplet (Schaum et al., 2018).

\section{Single-cell data analyses}

All analyses in this article were performed using the Seurat package version 3.0 in the $\mathrm{R}$ statistical language (Butler et al., 2018). Seurat objects were created from the aggregated datasets as matrix or text containing gene-by-cell expression data. Cells with expression of fewer than 200 genes and cells with more than $25 \%$ expression of mitochondrial genes were filtered out. The raw reads or UMI counts were log-normalized for downstream analysis. Highly variable genes were identified with the Seurat function of FindVaria-
bleFeatures. Genes with log-normalized expression values between 0.0125 and 3 and with a dispersion threshold 0.5 were considered variable. The ScaleData function was used to regress out cell-cell variation.

Principal component analysis (PCA) was performed for dimension reduction with the Seurat function of RunPCA. ElbowPlot function was used for determination of the numbers of principle components. The Seurat functions of FindNeighbors and FindClusters were used for clustering of cells. Dimension reduction was based on uniform manifold approximation and projection (UMAP), which was performed with the function of RunUMAP.

Genes significantly expressed in each cluster were screened by differential expression analysis (DEA) between cells inside and outside of the cluster with the Seurat function FindMarkers. Marker genes of each subpopulation used in heatmap were identified with the FindAllMarkers function.

Visualization of the cellar atlas was performed with Seurat function Dimplot. Visualization of the gene expression profiles was performed with Seurat function FeaturePlot and VlnPlot. Heatmap was performed with Seurat function DoHeatmap.

Integration of multiple single-cell datasets was based on identifying common gene-gene correlation patterns in different datasets. The identification of shared populations across datasets was performed with Seurat function FindIntegrationAnchors. The integration was performed with Seurat function IntegrateData.

\section{Code availability}

$\mathrm{R}$ scripts enabling the main steps of the analysis are available from the corresponding authors on reasonable request.

Compliance and ethics The author(s) declare that they have no conflict of interest.

Acknowledgements The authors acknowledge with thanks the $R$ script used for cellphone DB analysis provided by $\mathrm{Dr} N$. C. Henderson. This work was supported in part by the National Key Research and Development Program of China (2019YFA0802700, 2017YFA0103700) and the National Natural Science Foundation of China (91739301, 91339205, 81130005).

\section{References}

Aird, W.C. (2007a). Phenotypic heterogeneity of the endothelium. Circ Res 100, 158-173.

Aird, W.C. (2007b). Phenotypic heterogeneity of the endothelium. Circ Res 100, 174-190.

Armulik, A., Abramsson, A., and Betsholtz, C. (2005). Endothelial/pericyte interactions. Circ Res 97, 512-523.

Armulik, A., Genové, G., and Betsholtz, C. (2011). Pericytes: developmental, physiological, and pathological perspectives, problems, and promises. Dev Cell 21, 193-215.

Armulik, A., Genové, G., Mäe, M., Nisancioglu, M.H., Wallgard, E., Niaudet, C., He, L., Norlin, J., Lindblom, P., Strittmatter, K., et al. (2010). Pericytes regulate the blood-brain barrier. Nature 468, 557- 
561.

Arnold, T.D., Niaudet, C., Pang, M.F., Siegenthaler, J., Gaengel, K., Jung, B., Ferrero, G.M., Mukouyama, Y., Fuxe, J., Akhurst, R., et al. (2014). Excessive vascular sprouting underlies cerebral hemorrhage in mice lacking $\alpha$ V $\beta 8$-TGF $\beta$ signaling in the brain. Development 141, 44894499.

Augustin, H.G., and Koh, G.Y. (2017). Organotypic vasculature: From descriptive heterogeneity to functional pathophysiology. Science 357, eaal2379.

Bondjers, C., He, L., Takemoto, M., Norlin, J., Asker, N., Hellström, M., Lindahl, P., Betsholtz, C., Bondjers, C., He, L., et al. (2006). Microarray analysis of blood microvessels from PDGF-B and PDGF-R $\beta$ mutant mice identifies novel markers for brain pericytes. FASEB J 20, 17031705.

Bondjers, C., Kalén, M., Hellström, M., Scheidl, S.J., Abramsson, A., Renner, O., Lindahl, P., Cho, H., Kehrl, J., and Betsholtz, C. (2003). Transcription profiling of platelet-derived growth factor-B-deficient mouse embryos identifies RGS5 as a novel marker for pericytes and vascular smooth muscle cells. Am J Pathol 162, 721-729.

Borysova, L., Wray, S., Eisner, D.A., and Burdyga, T. (2013). How calcium signals in myocytes and pericytes are integrated across in situ microvascular networks and control microvascular tone. Cell Calcium 54, 163-174.

Boucher, J.M., Harrington, A., Rostama, B., Lindner, V., and Liaw, L. (2013). A receptor-specific function for Notch2 in mediating vascular smooth muscle cell growth arrest through cyclin-dependent kinase inhibitor 1B. Circ Res 113, 975-985.

Butler, A., Hoffman, P., Smibert, P., Papalexi, E., and Satija, R. (2018). Integrating single-cell transcriptomic data across different conditions, technologies, and species. Nat Biotechnol 36, 411-420.

Chen, J., Luo, Y., Hui, H., Cai, T., Huang, H., Yang, F., Feng, J., Zhang, J., and Yan, X. (2017). CD146 coordinates brain endothelial cell-pericyte communication for blood-brain barrier development. Proc Natl Acad Sci USA 114, E7622-E7631.

Chen, L., Li, X., Chen, M., Feng, Y., and Xiong, C. (2020). The ACE2 expression in human heart indicates new potential mechanism of heart injury among patients infected with SARS-CoV-2. Cardiovasc Res 116, $1097-1100$.

Chen, W.C.W., Baily, J.E., Corselli, M., Díaz, M.E., Sun, B., Xiang, G., Gray, G.A., Huard, J., and Péault, B. (2015). Human myocardial pericytes: multipotent mesodermal precursors exhibiting cardiac specificity. Stem Cells 33, 557-573.

Chou, J., Lin, J.H., Brenot, A., Kim, J., Provot, S., and Werb, Z. (2013). GATA3 suppresses metastasis and modulates the tumour microenvironment by regulating microRNA-29b expression. Nat Cell Biol 15, 201-213.

Christian, S., Winkler, R., Helfrich, I., Boos, A.M., Besemfelder, E., Schadendorf, D., and Augustin, H.G. (2008). Endosialin (Tem1) is a marker of tumor-associated myofibroblasts and tumor vessel-associated mural cells. Am J Pathol 172, 486-494.

Cooke, V.G., LeBleu, V.S., Keskin, D., Khan, Z., O'Connell, J.T., Teng, Y., Duncan, M.B., Xie, L., Maeda, G., Vong, S., et al. (2012). Pericyte depletion results in hypoxia-associated epithelial-to-mesenchymal transition and metastasis mediated by met signaling pathway. Cancer Cell 21, 66-81.

Daneman, R., Zhou, L., Kebede, A.A., and Barres, B.A. (2010). Pericytes are required for blood-brain barrier integrity during embryogenesis. Nature 468, 562-566.

Dermietzel, R., and Krause, D. (1991). Molecular anatomy of the bloodbrain-barrier as defined by immunocytochemistry. Int Rev Cytol 127, $57-109$.

Diaz-Flores, L., Gutierrez, R., Madrid, J. F., Varela, H., Valladares, F., Acosta, E., Martin-Vasallo, P., and Diaz-Flores, L., Jr. (2009). Pericytes. Morphofunction, interactions and pathology in a quiescent and activated mesenchymal cell niche. Histol Histopathol 24, 909-969.

Fantin, A., Herzog, B., Mahmoud, M., Yamaji, M., Plein, A., Denti, L., Ruhrberg, C., and Zachary, I. (2014). Neuropilin 1 (NRP1) hypomorphism combined with defective VEGF-A binding reveals novel roles for NRP1 in developmental and pathological angiogenesis. Development 141, 556-562.

Gao, Q., Zhang, J., Wang, X., Liu, Y., He, R., Liu, X., Wang, F., Feng, J., Yang, D., Wang, Z., et al. (2017). The signalling receptor MCAM coordinates apical-basal polarity and planar cell polarity during morphogenesis. Nat Commun 8, 15279.

Gerhardt, H., and Betsholtz, C. (2003). Endothelial-pericyte interactions in angiogenesis. Cell Tissue Res 314, 15-23.

Guimarães-Camboa, N., Cattaneo, P., Sun, Y., Moore-Morris, T., Gu, Y., Dalton, N.D., Rockenstein, E., Masliah, E., Peterson, K.L., Stallcup, W. B., et al. (2017). Pericytes of multiple organs do not behave as mesenchymal stem cells in vivo. Cell Stem Cell 20, 345-359.e5.

Hartmann, D.A., Underly, R.G., Grant, R.I., Watson, A.N., Lindner, V., and Shih, A.Y. (2015). Pericyte structure and distribution in the cerebral cortex revealed by high-resolution imaging of transgenic mice. Neurophotonics 2, 041402.

He, L., Vanlandewijck, M., Raschperger, E., Andaloussi Mäe, M., Jung, B., Lebouvier, T., Ando, K., Hofmann, J., Keller, A., and Betsholtz, C. (2016). Analysis of the brain mural cell transcriptome. Sci Rep 6, 35108 .

Hinkel, R., Howe, A., Renner, S., Ng, J., Lee, S., Klett, K., Kaczmarek, V., Moretti, A., Laugwitz, K.L., Skroblin, P., et al. (2017). Diabetes mellitus-induced microvascular destabilization in the myocardium. J Am Coll Cardiol 69, 131-143.

Huang, F.J., You, W.K., Bonaldo, P., Seyfried, T.N., Pasquale, E.B., and Stallcup, W.B. (2010). Pericyte deficiencies lead to aberrant tumor vascularizaton in the brain of the NG2 null mouse. Dev Biol 344, $1035-1046$.

Jung, J., Yang, K., Kim, H.J., Lee, Y.J., Kim, M., Choi, Y.H., and Kang, J.L. (2019). RhoA-dependent HGF and c-Met mediate Gas6-induced inhibition of epithelial-mesenchymal transition, migration, and invasion of lung alveolar epithelial cells. Biomolecules 9, 565 .

Kalucka, J., de Rooij, L.P.M.H., Goveia, J., Rohlenova, K., Dumas, S.J., Meta, E., Conchinha, N.V., Taverna, F., Teuwen, L.A., Veys, K., et al. (2020). Single-cell transcriptome atlas of murine endothelial cells. Cell 180, 764-779.e20.

Kato, K., Diéguez-Hurtado, R., Park, D.Y., Hong, S.P., Kato-Azuma, S., Adams, S., Stehling, M., Trappmann, B., Wrana, J.L., Koh, G.Y., et al. (2018). Pulmonary pericytes regulate lung morphogenesis. Nat Commun 9, 2448.

Keller, A., Westenberger, A., Sobrido, M.J., García-Murias, M., Domingo, A., Sears, R.L., Lemos, R.R., Ordoñez-Ugalde, A., Nicolas, G., da Cunha, J.E.G., et al. (2013). Mutations in the gene encoding PDGF-B cause brain calcifications in humans and mice. Nat Genet $45,1077-$ 1082 .

Kovac, A., Erickson, M.A., and Banks, W.A. (2011). Brain microvascular pericytes are immunoactive in culture: cytokine, chemokine, nitric oxide, and LRP-1 expression in response to lipopolysaccharide. J Neuroinflamm 8, 139.

Kurz, H., Fehr, J., Nitschke, R., and Burkhardt, H. (2008). Pericytes in the mature chorioallantoic membrane capillary plexus contain desmin and $\alpha$-smooth muscle actin: relevance for non-sprouting angiogenesis. Histochem Cell Biol 130, 1027-1040.

MacDonald, T.J., Brown, K.M., LaFleur, B., Peterson, K., Lawlor, C., Chen, Y., Packer, R.J., Cogen, P., and Stephan, D.A. (2001). Expression profiling of medulloblastoma: PDGFRA and the RAS/MAPK pathway as therapeutic targets for metastatic disease. Nat Genet 29, 143-152.

Mathiisen, T.M., Lehre, K.P., Danbolt, N.C., and Ottersen, O.P. (2010). The perivascular astroglial sheath provides a complete covering of the brain microvessels: an electron microscopic 3D reconstruction. Glia 58, 1094-1103.

Matsuda, S., Matsuda, Y., and D'Adamio, L. (2009). CD74 interacts with APP and suppresses the production of A $\beta$. Mol Neurodegener 4, 41.

Nees, S., Juchem, G., Eberhorn, N., Thallmair, M., Förch, S., Knott, M., Senftl, A., Fischlein, T., Reichart, B., and Weiss, D.R. (2012). Wall structures of myocardial precapillary arterioles and postcapillary 
venules reexamined and reconstructed in vitro for studies on barrier functions. Am J Physiol-Heart Circulat Physiol 302, H51-H68.

Nehls, V., Denzer, K., and Drenckhahn, D. (1992). Pericyte involvement in capillary sprouting during angiogenesis in situ. Cell Tissue Res 270, 469-474.

Nolan, D.J., Ginsberg, M., Israely, E., Palikuqi, B., Poulos, M.G., James, D., Ding, B.S., Schachterle, W., Liu, Y., Rosenwaks, Z., et al. (2013). Molecular signatures of tissue-specific microvascular endothelial cell heterogeneity in organ maintenance and regeneration. Dev Cell 26, 204-219.

O'Keeffe, M.B., Devlin, A.H., Burns, A.J., Gardiner, T.A., Logan, I.D., Hirst, D.G., and McKeown, S.R. (2008). Investigation of pericytes, hypoxia, and vascularity in bladder tumors: association with clinical outcomes. Oncol Res 17, 93-101.

Ozerdem, U., Grako, K.A., Dahlin-Huppe, K., Monosov, E., and Stallcup, W.B. (2001). NG2 proteoglycan is expressed exclusively by mural cells during vascular morphogenesis. Dev Dyn 222, 218-227.

Paik, D.T., Tian, L., Lee, J., Sayed, N., Chen, I.Y., Rhee, S., Rhee, J.W., Kim, Y., Wirka, R.C., Buikema, J.W., et al. (2018). Large-scale singlecell RNA-Seq reveals molecular signatures of heterogeneous populations of human induced pluripotent stem cell-derived endothelial cells. Circ Res 123, 443-450.

Pantouris, G., Ho, J., Shah, D., Syed, M.A., Leng, L., Bhandari, V., Bucala, R., Batista, V.S., Loria, J.P., and Lolis, E.J. (2018). Nanosecond dynamics regulate the MIF-induced activity of CD74. Angew Chem Int Ed 57, 7116-7119.

Peppiatt, C.M., Howarth, C., Mobbs, P., and Attwell, D. (2006). Bidirectional control of CNS capillary diameter by pericytes. Nature 443, 700-704.

Potente, M., and Mäkinen, T. (2017). Vascular heterogeneity and specialization in development and disease. Nat Rev Mol Cell Biol 18, 477-494.

Ramachandran, P., Dobie, R., Wilson-Kanamori, J.R., Dora, E.F., Henderson, B.E.P., Luu, N.T., Portman, J.R., Matchett, K.P., Brice, M., Marwick, J.A., et al. (2019). Resolving the fibrotic niche of human liver cirrhosis at single-cell level. Nature 575, 512-518.

Rensen, S.S.M., Doevendans, P.A.F.M., and van Eys, G.J.J.M. (2007). Regulation and characteristics of vascular smooth muscle cell phenotypic diversity. Neth Heart J 15, 100-108.

Ricard, N., Tu, L., Le Hiress, M., Huertas, A., Phan, C., Thuillet, R., Sattler, C., Fadel, E., Seferian, A., Montani, D., et al. (2014). Increased pericyte coverage mediated by endothelial derived Fgf-2 and Il-6 is a source of smooth muscle-like cells. Am J Resp Crit Care 189, A4823.

Saban, M.R., Backer, J.M., Backer, M.V., Maier, J., Fowler, B., Davis, C. A., Simpson, C., Wu, X.R., Birder, L., Freeman, M.R., et al. (2008).
VEGF receptors and neuropilins are expressed in the urothelial and neuronal cells in normal mouse urinary bladder and are upregulated in inflammation. Am J Physiol-Renal Physiol 295, F60-F72.

Schaum, N., Karkanias, J., Neff, N.F., May, A.P., Quake, S.R., Wyss-Coray, T., Darmanis, S., Batson, J., and Botvinnik, O. (2018). Single-cell transcriptomics of 20 mouse organs creates a Tabula Muris. Nature 562, 367-372.

Schlingemann, R.O., Oosterwijk, E., Wesseling, P., Rietveld, F.J.R., and Ruiter, D.J. (1996). Aminopeptidase A is a constituent of activated pericytes in angiogenesis. J Pathol 179, 436-442.

Shepro, D., and Morel, N.M.L. (1993). Pericyte physiology. FASEB J 7, 1031-1038.

Shibata, T., Makino, A., Ogata, R., Nakamura, S., Ito, T., Nagata, K., Terauchi, Y., Oishi, T., Fujieda, M., Takahashi, Y., et al. (2020). Respiratory syncytial virus infection exacerbates pneumococcal pneumonia via Gas6/Axl-mediated macrophage polarization. J Clin Invest 130, 3021-3037.

Sims, D., Horne, M.M., Creighan, M., and Donald, A. (1994). Heterogeneity of pericyte populations in equine skeletal muscle and dermal microvessels: a quantitative study. Anatom Histol Embryol 23, 232-238.

Stratman, A.N., Schwindt, A.E., Malotte, K.M., and Davis, G.E. (2010). Endothelial-derived PDGF-BB and HB-EGF coordinately regulate pericyte recruitment during vasculogenic tube assembly and stabilization. Blood 116, 4720-4730.

Teichert, M., Milde, L., Holm, A., Stanicek, L., Gengenbacher, N., Savant, S., Ruckdeschel, T., Hasanov, Z., Srivastava, K., Hu, J., et al. (2017). Pericyte-expressed Tie2 controls angiogenesis and vessel maturation. Nat Commun 8, 16106.

Vento-Tormo, R., Efremova, M., Botting, R.A., Turco, M.Y., Vento-Tormo, M., Meyer, K.B., Park, J.E., Stephenson, E., Polański, K., Goncalves, A., et al. (2018). Single-cell reconstruction of the early maternal-fetal interface in humans. Nature 563, 347-353.

Williams, M.J., Sugatani, T., Agapova, O.A., Fang, Y., Gaut, J.P., Faugere, M.C., Malluche, H.H., and Hruska, K.A. (2018). The activin receptor is stimulated in the skeleton, vasculature, heart, and kidney during chronic kidney disease. Kidney Int 93, 147-158.

Winkler, E.A., Birk, H., Burkhardt, J.K., Chen, X., Yue, J.K., Guo, D., Rutledge, W.C., Lasker, G.F., Partow, C., Tihan, T., et al. (2018). Reductions in brain pericytes are associated with arteriovenous malformation vascular instability. J Neurosurg 129, 1464-1474.

Wu, C., Thalhamer, T., Franca, R.F., Xiao, S., Wang, C., Hotta, C., Zhu, C., Hirashima, M., Anderson, A.C., and Kuchroo, V.K. (2014). Galectin-9CD44 interaction enhances stability and function of adaptive regulatory T cells. Immunity 41, 270-282.

\section{SUPPORTING INFORMATION}

The supporting information is available online at https://doi.org/10.1007/s11427-020-1823-2. The supporting materials are published as submitted, without typesetting or editing. The responsibility for scientific accuracy and content remains entirely with the authors. 